REVIEW

\title{
Chondrule formation and evolution of the early solar system*
}

\author{
Shogo TACHIBANA \\ Department of Earth and Planetary Science, Graduate School of Science, \\ The University of Tokyo, 7-3-1 Hongo, Tokyo 113-0033, Japan
}

\begin{abstract}
Chondrules are millimeter to sub-millimeter size silicate spherules that formed during localized and transient high-temperature events in the early solar system. Although it is not yet understood how chondrules formed, recent studies have provided important clues in understanding the physical conditions of chondrule formation. In this paper I review recent developments in studies of chondrules to provide constraints on high-temperature processes during the evolution of protoplanetary disks, including: (1) the timing and duration of the chondruleforming high-temperature period, based on long-lived and short-lived chronometers; (2) the thermal history of chondrule melts during each chondrule-forming event, as constrained from laboratory experiments and petrological and isotopic studies of chondrules, including shock-wave heating as a plausible heat source for chondrule formation; and (3) sources for shock waves and chondrule-forming regions in the protoplanetary disk. Given our current knowledge, I provide the following possible answers to the above four points: (1) the period of chondrule formation began shortly after the formation of calcium-aluminum-rich inclusions and lasted for a few million years; (2) chondrule precursors were heated at a rate of $>10^{4} \mathrm{~K} / \mathrm{h}$ in the temperature range of $\sim 1400-1600 \mathrm{~K}$, and melt droplets were cooled from a peak temperature of $\sim 1800-2200 \mathrm{~K}$ at a rate of $\sim 5$ $1000 \mathrm{~K} / \mathrm{h}$; (3) high-velocity shock waves $(>20 \mathrm{~km} / \mathrm{s})$ in a low-density gas region $\left(<10^{19}\right.$ particles $\left./ \mathrm{m}^{3}\right)$ may be appropriate for localized transient heating events associated with chondrule formation; and (4) $\mathrm{X}^{-}$-ray flares from the young Sun in its T-Tauri stage might be the source of the shock waves.
\end{abstract}

Keywords: Chondrule, Radionuclide, Chronology, Thermal history, Shock-wave heating, X-ray flare, Early solar system

\section{INTRODUCTION}

The parent molecular cloud of the Sun collapsed to form the infant Sun and a surrounding protoplanetary disk at $\sim 4.57 \mathrm{Ga}$. Dust particles in the protoplanetary disk were annealed or evaporated due to heating during the active stage of the protoplanetary disk, resulting in changes to the crystal structure and chemical and isotopic composition of dust particles. During later stages of the disk evolution, dust particles accumulated to form planetesimals, which later formed diverse planets. Broken pieces of planetesimals have fallen on Earth as meteorites. More than $80 \%$ of the meteorites, which are called chondrites, did not experience significant melting when within their parent bodies, and appear to be heterogeneous aggregates of components that formed by various processes in the protoplanetary disk and on their parent bodies. Some components in chondrites, especially within the least meta-

\footnotetext{
* Invited review based on the lecture at the 2004 Symposium of the Mineralogical Society of Japan "The Frontiers of Science for Primitive Solar System Materials: The Role of Mineral Science".

S. Tachibana, tachi@eps.s.u-tokyo.ac.jp Corresponding author
}

morphosed chondrites, thus record the evolution of solid material in the early solar system. The most fascinating components in chondrites are chondrules, which are major constituents of chondrites and are millimeter to sub-millimeter sized spherules consisting of silicate phenocrysts, glassy mesostasis, and a small fraction of opaque phases (Fig. 1). Their spherical shapes and internal textures suggest that chondrules were once molten, even during the early solar system, and were cooled relatively rapidly. The presence of relict grains, such as a forsteritic olivine grain in an iron-rich olivine phenocryst (Fig. 1a), indicates that chondrules may have formed by repeated melting of solid precursors (Nagahara, 1981; Rambaldi, 1981; Jones, 1996). These inferred conditions for chondrule formation suggest that localized transient high-temperature events occurred repeatedly in the early solar system. Thus, to understand the evolution of both planetary material and the protoplanetary disk, it is important to understand when the period of chondrule formation started, how long it lasted, the thermal history of chondrules during each heating event, the heat source for melting, and where chondrule formation occurred. 
In this paper, I review recent progress in the field of planetary material science, especially studies of chondrules, some of which may provide clues to answer the above questions. The paper is organized as follows. In the following section, I review the timing and duration of the chondrule-forming period based on long-lived and shortlived radionuclide chronometers. In the third section, I review the thermal history of chondrules during each chondrule-forming event, and possible heat sources for chondrule formation that are consistent with the inferred thermal history. Cooling histories of chondrule melt droplets have been revealed by experimental studies that reproduced the textures of chondrules, although heating histories of chondrule precursors have yet to be discussed. A recent study of the sulfur isotopic compositions of chondrule troilites may provide quantitative constraints on the heating rates of chondrule precursors. Possible heating mechanisms and sites of chondrule-formation will be discussed based on the estimated thermal history of chondrules.

\section{TIMING AND DURATION OF CHONDRULE- FORMING EVENTS}

Radionuclides decay into stable daughter nuclides at specific decay rates. Radionuclides with half-lives $\left(t_{1 / 2}\right)$ that are comparable to or longer than the age of the solar system still exist in the solar system. By using long-lived radionuclides such as ${ }^{40} \mathrm{~K}\left(t_{1 / 2}=1.3 \mathrm{Gyr},{ }^{87} \mathrm{Rb}(48.8 \mathrm{Gyr})\right.$, ${ }^{147} \mathrm{Sm}$ (106 Gyr), ${ }^{187} \operatorname{Re}(45.6 \mathrm{Gyr}),{ }^{232} \mathrm{Th}$ (14 Gyr), ${ }^{235} \mathrm{U}$ (0.704 Gyr), and ${ }^{238} \mathrm{U}(4.47 \mathrm{Gyr})$, the absolute age of an object can be determined from the abundance of the parent radionuclide when the object formed and the abundance of the daughter nuclide produced by decay since the time of formation. However, it is quite difficult to precisely measure the small difference in isotopic compositions required to obtain the time difference of events that occurred within a few million years of each other in the early solar system.

Radionuclides with short half-lives ( $\leq 100 \mathrm{Myr}$ ) are no longer observed because they have almost completely decayed. Isotopic analyses of meteorites reveal that the following short-lived radionuclides once existed in the early solar system: ${ }^{41} \mathrm{Ca}\left(t_{1 / 2}=0.1 \mathrm{Myr}\right),{ }^{36} \mathrm{Cl}(0.3 \mathrm{Myr})$, ${ }^{26} \mathrm{Al}$ (0.73 Myr), ${ }^{60} \mathrm{Fe}$ (1.49 Myr), ${ }^{10} \mathrm{Be}$ (1.5 Myr), ${ }^{53} \mathrm{Mn}(3.7$ $\mathrm{Myr}),{ }^{107} \mathrm{Pd}(6.5 \mathrm{Myr}),{ }^{182} \mathrm{Hf}$ (9 Myr), ${ }^{129} \mathrm{I}(15.7 \mathrm{Myr}),{ }^{92} \mathrm{Nb}$ (36 Myr), ${ }^{244} \mathrm{Pu}$ (82 Myr), and ${ }^{146} \mathrm{Sm}$ (103 Myr) (McKeegan and Davis, 2003; Kita et al., 2005a and references therein). Beryllium-7, with a half-life of 53 days, might also have existed (Chaussidon et al., 2002). The presence of short-lived radionuclides in the early solar system can be confirmed by an excess of daughter nuclides $(D)$ in an object that correlates with a given amount of the parent element. The abundance of $D$ in an object is the sum of the radiogenic component $\left(D^{*}\right)$ that decayed from a shortlived radionuclide $(P)$ and the nonradiogenic component $\left(D_{0}\right)$, i.e., $D=D_{0}+D^{*}$. The ratio of $D$ to the stable nuclide of the same element $\left(D_{s}\right)$ can be expressed by the ratio of the amount of the stable nuclide of the parent element $\left(P_{s}\right)$ to $D_{s}$ and the abundance of the short-lived radionuclide relative to the stable nuclide of the same element $\left(\left(P / P_{s}\right)_{0}\right)$ at the time of the last isotopic equilibration:

$$
\frac{D}{D_{s}}=\frac{D_{0}+D^{*}}{D_{s}}=\frac{D_{0}+P_{0}}{D_{s}}=\left(\frac{D}{D_{s}}\right)_{0}+\left(\frac{P}{P_{s}}\right)_{0}\left(\frac{P_{s}}{D_{s}}\right),
$$

where $D^{*}=P_{0}$ because $P_{0}$ has been completely converted to $D^{*}$. Thus, the linear regression of data sets for $D / D_{s}$ and $P_{\mathrm{s}} / D_{s}$ from the object gives $\left(P / P_{s}\right)_{0}$ at the time of the last isotopic equilibration. In principle, if two objects $A$ and $B$ formed from an isotopically homogeneous reservoir at a time interval of $\Delta t, \Delta t$ can be obtained from the difference between the $\left(P / P_{s}\right)_{0}$ ratio of the two objects:

$$
\Delta t=\ln \frac{\left(P / P_{s}\right)_{0, A}}{\left(P / P_{s}\right)_{0, B}} \cdot \frac{\Delta t_{1 / 2}}{\ln 2}
$$

where $\left(P / P_{s}\right)_{0, A}$ and $\left(P / P_{s}\right)_{0, B}$ are $\left(P / P_{s}\right)_{0}$ for the objects $A$ and $B$, respectively.

Although short-lived chronometers can only be used to estimate the $\Delta t$ (relative ages) of different objects, the precision of $\Delta t$ can be as fine as $<1 \mathrm{Myr}$ due to the short half-lives of the radionuclides. Thus, short-lived radionuclides are potentially useful chronometers for events that occurred in the protoplanetary disk. It should be noted here that for a short-lived radionuclide to act as a chronometer, three conditions must be met: (i) the abundance of the radionuclide relative to stable isotopes of the same element must be constant throughout the object at the time of formation; (ii) chemical fractionation must occur at the time of formation to produce minerals with different relative abundances of parent and daughter elements; and (iii) once formed, the object must remain chemically closed and undisturbed, permitting the daughter isotope to accumulate by radioactive decay in amounts proportional to the abundances of the parent element. If these conditions are met, then the abundance of the daughter isotope will correlate with the abundance of the parent element. More details of radionuclides in the early solar system and chronometers based on radionuclides are described by McKeegan and Davis (2003) and Kita et al. (2005a). 


\section{Relative ages of chondrules based on the ${ }^{26} \mathrm{Al}-{ }^{26} \mathrm{Mg}$ short-lived chronometer}

Aluminum-26 is a short-lived radionuclide that decays into ${ }^{26} \mathrm{Mg}$ by $\beta^{-}$emission, with a half-life of $0.73 \mathrm{Myr}$. Evidence of live ${ }^{26} \mathrm{Al}$ in the early solar system was found in calcium-aluminum-rich inclusions (CAIs), the oldest solid objects known from the early solar system, from the Allende meteorite (e.g., Lee et al., 1976). A number of studies of ${ }^{26} \mathrm{Al}$ in CAIs have shown that many CAIs have an initial ${ }^{26} \mathrm{Al} /{ }^{27} \mathrm{Al}$ ratio of $5 \times 10^{-5}$, which has been interpreted as the canonical value for the early solar system (see MacPherson et al., 1995 and references therein). Recently, an initial ${ }^{26} \mathrm{Al} /{ }^{27} \mathrm{Al}$ ratio of $(6-7) \times 10^{-5}$, which is higher than the canonical value, has been reported for several CAIs analyzed by multicollector inductively coupled mass spectrometry (MC-ICPMS) (Galy et al., 2000; Galy et al., 2004; Young et al., 2005). This may indicate that formation of CAIs began $\sim 0.3 \mathrm{Myr}$ earlier than closure of the ${ }^{26} \mathrm{Al}-{ }^{26} \mathrm{Mg}$ systems in most CAIs with a $\left({ }^{26} \mathrm{Al} /{ }^{27} \mathrm{Al}\right)_{0}$ value of $5 \times 10^{-5}$ (Young et al., 2005); this should therefore be investigated intensively in future studies.

Studies of ${ }^{26} \mathrm{Al}^{-26} \mathrm{Mg}$ systems in chondrules have concentrated on rare $\mathrm{Al}$-rich chondrules, because high $\mathrm{Al} / \mathrm{Mg}$ phases are necessary to resolve excess ${ }^{26} \mathrm{Mg}$ (e.g., Russel et al., 1996; Huss et al., 2001). Excess ${ }^{26} \mathrm{Mg}$, correlated with the $\mathrm{Al} / \mathrm{Mg}$ ratio, from common ferromagnesian chondrules was first found by Kita et al. (2000) via ion microprobe analyses of ferromagnesian chondrules in Semarkona (LL3.0), one of the least metamorphosed ordinary chondrites. The obtained initial ${ }^{26} \mathrm{Al} /{ }^{27} \mathrm{Al}$ ratios for the chondrules are in the range $0.87-0.47 \times 10^{-5}$, which is smaller than the canonical value for CAIs. Mostefaoui et al. (2002) reported further resolvable ${ }^{26} \mathrm{Mg}$ excesses in 11 ferromagnesian chondrules in Bishunpur (LL3.1). Similar results were obtained from chondrules in Semarkona and Bishunpur by McKeegan et al. (2000), and chondrules from Krymka (LL3.1) by Tomomura et al. (2004) and Kita et al. (2005b). Ferromagnesian chondrules from Y81020 (CO3.0) also show excess ${ }^{26} \mathrm{Mg}$, and the obtained range of initial ${ }^{26} \mathrm{Al} /{ }^{27} \mathrm{Al}$ ratios is comparable to that for chondrules from ordinary chondrites (Yurimoto and Wasson, 2003; Kunihiro et al., 2004; Kurahashi et al., 2004); this suggests that chondrules from both ordinary and carbonaceous chondrites formed contemporaneously.

If ${ }^{26} \mathrm{Al}$ was homogeneously distributed in the solar system (or at least in the $\mathrm{CAI}^{-}$and chondrule-forming region) with the canonical value, which is one of the conditions required for a short-lived chronometer, the range of initial $\left({ }^{26} \mathrm{Al} /{ }^{27} \mathrm{Al}\right)$ ratios of those chondrules would correspond to a time interval that is $\sim 1-3 \mathrm{Myr}$ after CAI formation. The data sets for chondrules with excess ${ }^{26} \mathrm{Mg}$ show that the age-distribution of chondrules has a peak at $\sim 2$ Myr [see Fig. 4 in Kita et al. (2005)]. Because Semarkona, Bishunpur, Krymka, and Y81020 are among the least metamorphosed ordinary and carbonaceous chondrites, the observed ranges in initial $\left({ }^{26} \mathrm{Al} /{ }^{27} \mathrm{Al}\right)$ ratios have chronological significance for chondrule formation. The observed ratios suggest that chondrule-forming events lasted at least for a few Myr at almost the same time within the formation regions of ordinary and carbonaceous chondrites and that chondrules with older ages did not experience the heating events that formed younger chondrules (Mostefaoui et al., 2002; Kurahashi et al., 2004; Kita et al., 2005a).

Such an age distribution of chondrules may correlate with the history of high-temperature events in the early solar system. However, some chondrules contain relict grains derived from earlier generations of chondrules and were not melted during heating; this indicates that chondrules were heated repeatedly (e.g., Nagahara 1981; Rambaldi, 1981; Jones 1996). Initial ${ }^{26} \mathrm{Al} /{ }^{27} \mathrm{Al}$ ratios obtained from Al-rich minerals or mesostasis by ion microprobe probably record the last heating event for individual chondrules, as $\mathrm{Al}$-rich phases would have melted readily and their ${ }^{26} \mathrm{Al}-{ }^{26} \mathrm{Mg}$ systems would be reset by heating events. Thus, the age distribution of chondrules determined by ${ }^{26} \mathrm{Al}$ chronometry may record a peak at a younger age (i.e., longer time-interval from CAI formation) than the frequency distribution of high-temperature events (Nakamoto et al., 2005a).

Recent studies of Allende (CV3) chondrules using high-precision inductively coupled plasma mass spectrometry (ICP-MS) have shown that some chondrules have initial ${ }^{26} \mathrm{Al} /{ }^{27} \mathrm{Al}$ ratios close to the canonical value, suggesting that such chondrules formed at the same time as CAIs (Bizzarro et al., 2004). Considering that these initial ${ }^{26} \mathrm{Al} /{ }^{27} \mathrm{Al}$ ratios were not obtained for $\mathrm{Al}$-rich phases, the initial ${ }^{26} \mathrm{Al} /{ }^{27} \mathrm{Al}$ ratios obtained for bulk chondrules by ICP-MS may not record the time of the last melting event of chondrules, but represent the time of chondrule-precursor (silicate dust) formation (see also Fig. 5 in Kita et al., 2005a). Because measurements of Al-rich phases in chondrules can determine only the timing of the last melting events of chondrules, studies on the initial ${ }^{26} \mathrm{Al} /{ }^{27} \mathrm{Al}$ ratios for bulk chondrules are important in understanding when chondrule formation began.

It has been pointed out that the ${ }^{26} \mathrm{Al}$ ages of chondrules from unequilibrated ordinary chondrites may correlate with the bulk compositions of chondrules, especially $\mathrm{Si} / \mathrm{Mg}$ ratios (Mostefaoui et al., 2002; Tachibana et al., 2003). Younger chondrules tend to contain more $\mathrm{Si}$ and volatile elements, which has been interpreted by Tachibana et al. (2003) to result from partial evaporation 
and recondensation of these elements, accompanied by partial separation of chondrules from the chondrule-forming region. It appears that $\mathrm{FeO}$-rich chondrules from carbonaceous chondrites are younger than $\mathrm{MgO}$-rich chondrules (Kunihiro et al., 2003; Kurahashi et al., 2004). Although these correlations may imply that chemical evolution of planetary materials occurred in the chondruleforming epoch, there are only limited data of both bulk compositions and ${ }^{26} \mathrm{Al}$ ages; further investigations are therefore required.

\section{Absolute ages of chondrules based on the $\mathrm{Pb}-\mathrm{Pb}$ long- lived chronometer}

Amelin et al. (2002) determined high-precision $\mathrm{Pb}-\mathrm{Pb}$ ages of CAIs from Efremovka (CV3) and chondrules from Acfer $059(\mathrm{CR})$ of $4.5672( \pm 0.0006) \mathrm{Ga}$ and 4.5647 $( \pm 0.0006) \mathrm{Ga}$, respectively. Amelin et al. (2004) and Amelin and Krot (2005) further reported absolute ages of chondrules from Allende, Gujba (CB), and Hammadah al Hamra $237(\mathrm{CB})$ of $4.5667( \pm 0.0010) \mathrm{Ga}, 4.5627( \pm$ $0.0005) \mathrm{Ga}$, and $4.5628( \pm 0.0009) \mathrm{Ga}$, respectively. Considering that chondrules from CB chondrites may have been produced by impact-related processes on the parent body and may not be products in the protoplanetary disk (Rubin et al., 2003), the difference between the $\mathrm{Pb}-\mathrm{Pb}$ ages of CAIs and chondrules suggest that chondrule formation started contemporaneously with or shortly after CAI formation and lasted for $\sim 2$ Myr. Petrographical evidence from CAI-bearing chondrules (Krot et al., 2005) and chondrule-bearing CAIs (Itoh and Yurimoto, 2003; Krot et al., 2005) supports these dating results. The above conclusions appear to be consistent with the history of high-temperature chondrule-forming events estimated from the ${ }^{26} \mathrm{Al}{ }^{-26} \mathrm{Mg}$ chronometer, which in turn supports the proposed homogeneous distribution of $\mathrm{Al}$ isotopes in the early solar system.

\section{The evolutional stage of the protoplanetary disk as a time of chondrule formation}

The timing and duration of chondrule-forming high-temperature events in the early solar system, as inferred from short-lived and long-lived chronometers, is as follows. Chondrule formation started shortly after or contemporaneously with the formation of the oldest solid objects (CAIs) and would have lasted for a few Myr, indicating that localized transient energetic events occurred repeatedly and continually in the early solar system. Although it is not possible at present to directly correlate the chondrule formation period with the evolutional stage of the protoplanetary disk, high-temperature events could have occurred during the active stage of the protoplanetary disk (classical T-Tauri stage), which typically lasts for 1-3 Myr. During the active stage of the protoplanetary disk, material in the disk accreted onto the proto-Sun through the disk and only minimal planetesimal formation occurred. Chondrules thus may have been produced continuously throughout the active stage of the protoplanetary disk until the disk evolved to the next passive stage, at which point planetesimal formation began.

\section{THERMAL HISTORY OF EACH CHONDRULE- FORMING EVENT}

Chondrules were formed by the melting and subsequent cooling of solid precursors. However, the heating mechanism for chondrules is not yet clearly understood. To clarify transient energetic events in the early solar system responsible for chondrule formation, it is therefore important to quantitatively understand the heating and cooling conditions of chondrules.

\section{Peak temperatures and cooling rates of chondrule melt}

A large number of experimental studies reveal that peak temperatures and cooling rates of chondrule melt droplets are important factors in determining chondrule texture (Tsuchiyama et al., 1980; Hewins, 1988; Lofgren, 1996; Hewins and Connolly, 1996; Jones et al., 2000; Desch and Connolly, 2002 and references therein).

Dynamic crystallization experiments have shown that porphyritic textures (Fig. 1a-d) are produced by nucleation on relict crystals that represent incomplete melting of precursor crystals, while barred (Fig. 1e) and radial (Fig. 1f) textures are produced when precursors are heated and melted entirely above their liquidus temperatures. The range of peak temperatures of chondrule formation has been estimated to be $\sim 1800-2200 \mathrm{~K}$.

The crystallization timescale of chondrule melt droplets is defined by cooling rates. Laboratory experiments and observations of phenocryst microstructures show that cooling rates of $\sim 5-1000 \mathrm{~K} / \mathrm{h}$ are required to reproduce the textural and chemical signatures of natural chondrules, including the sizes and chemical zoning of phenocrysts. Estimated cooling rates are much slower than those for radiative cooling of melt droplets to free space $\left(\sim 10^{6}\right.$ $\mathrm{K} / \mathrm{h}$ ); there must therefore be a mechanism that controls the cooling rate of chondrules.

Some chondrules contain volatile elements such as alkali elements and sulfur. Experimental studies on the evaporation of volatiles from chondrule melts (e.g., Tsuchiyama et al., 1981; Yu et al., 1996; Yu and Hewins, 

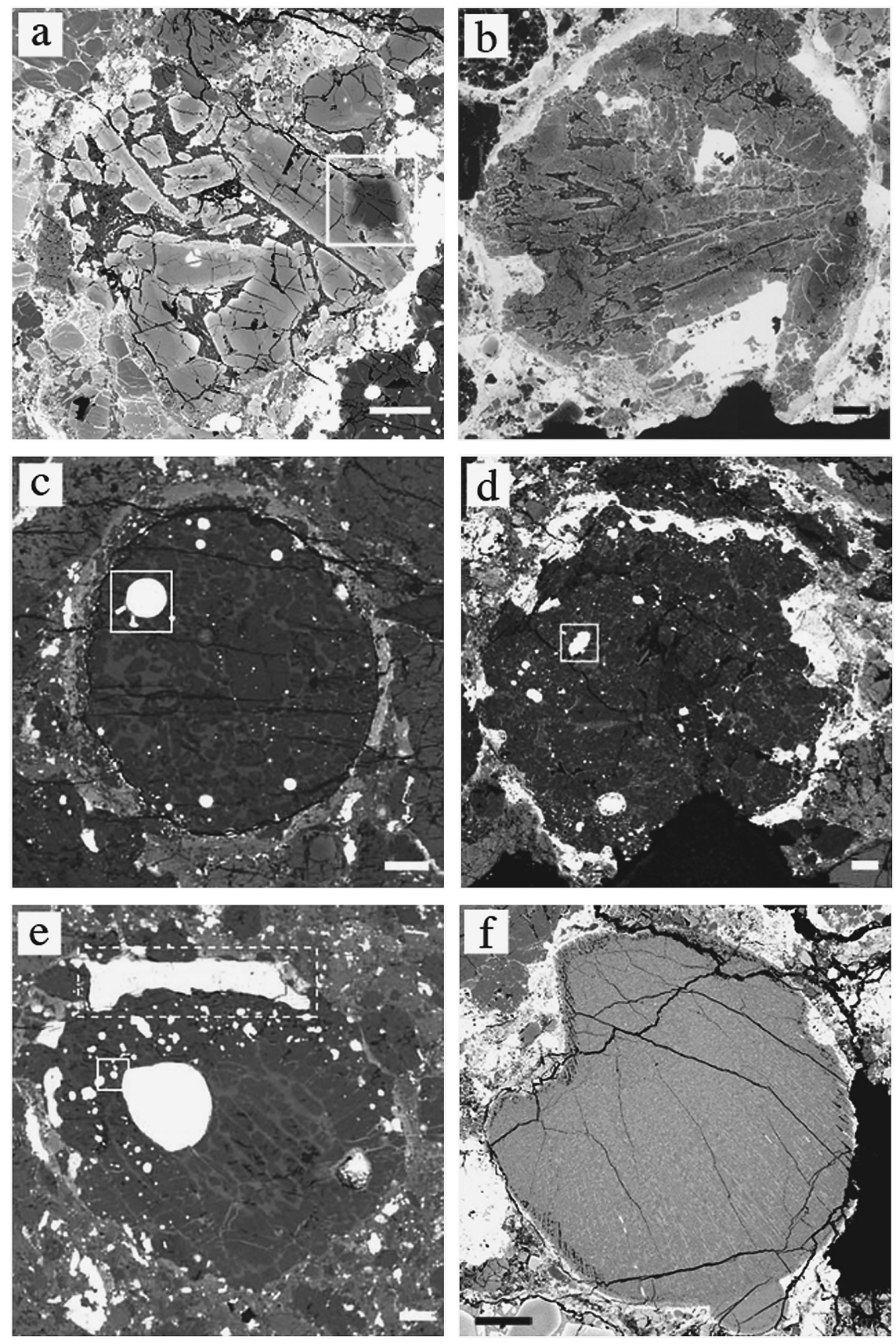

Figure 1. Back-scattered electron images of ferromagnesian chondrules. Scale bars are $100 \mu \mathrm{m}$. (a) $\mathrm{FeO}$-rich olivine-pyroxene porphyritic chondrule from Bishunpur (LL3.1) (B2-C12 in Mostefaoui et al. (2002) and Tachibana et al. (2003)). The initial $\left({ }^{26} \mathrm{Al} /{ }^{27} \mathrm{Al}\right)_{0}$ is $4.5( \pm 2.1) \times 10^{-6}$ (Mostefaoui et al., 2002). A relict forsteritic olivine grain is shown in the white box. (b) $\mathrm{FeO}$-rich olivine-pyroxene porphyritic chondrule from Krymka (LL3.1), with an initial $\left({ }^{26} \mathrm{Al} /{ }^{27} \mathrm{Al}\right)_{0}$ of $6.1( \pm 1.7) \times$ $10^{-6}$ (K27 in Tomomura et al. (2004) and Kita et al. (2005b)). (c) MgO-rich olivine porphyritic chondrule from Bishunpur (LL3.1). B1-CH15 in Tachibana and Huss (2005). The sulfur isotopic composition of troilite shown inside the white box was measured (see Fig. 2a). (d) $\mathrm{MgO}$-rich olivine porphyritic chondrule from Semarkona (LL3.0). S1-CH2 in Tachibana and Huss (2005). The sulfur isotopic composition of troilite shown inside the white box was measured (see Fig. 2b). (e) $\mathrm{MgO}$-rich barred olivine chondrule with a thick olivine armor from Bishunpur (LL3.1). B1-CH18 in Tachibana and Huss (2005). The sulfur isotopic composition of troilite shown inside the white box was measured (see Fig. 2c). The troilite shown inside the dashed box was measured as a reference. (f) $\mathrm{FeO}$-rich fine-grained radiating pyroxene chondrule from Semarkona (LL3.0), in which clear evidence of ${ }^{60} \mathrm{Fe}$ was found $\left[\left({ }^{60} \mathrm{Fe} /{ }^{56} \mathrm{Fe}\right)_{0}=2.3( \pm 1.0) \times\right.$ $10^{-7}$; SMK2-1 in Tachibana et al. (2006)].
1998) suggest that faster cooling rates $(\sim 5000 \mathrm{~K} / \mathrm{h})$ may be required for the initial cooling stage (Yu et al., 1996; Yu and Hewins, 1998). However, it should be noted that volatile elements, once evaporated, may have recondensed onto chondrule melts during cooling (e.g., Nagahara and Ozawa, 2000; Ozawa and Nagahara, 2001). This suggests that the retention of volatile elements does not necessarily represent only minor evaporation of these elements due to rapid cooling. Sulfur is expected to behave differently than alkali elements during chondrule melting, as will be shown later in the text, and retention of sulfur in chondrules may place constraints on the thermal history of chondrules.

\section{Heating rates of chondrule melt}

Although there are no quantitative constraints on the heating conditions of chondrule precursors, Tachibana and Huss (2005) made a quantitative estimate of heating rates of chondrule precursors $\left(>10^{4} \mathrm{~K} / \mathrm{h}\right)$ from the sulfur isotopic compositions of troilites located inside and outside of chondrules in Semarkona (LL3.0) and Bishunpur (LL3.1). 

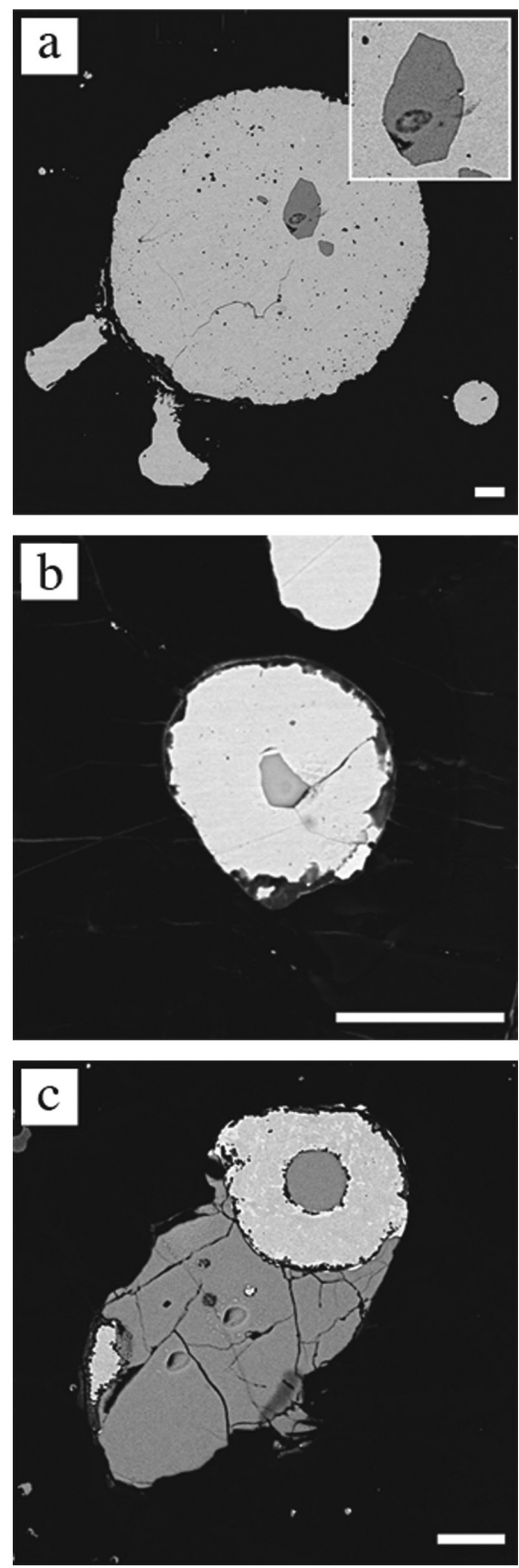

Figure 2. Back-scattered electron images of troilites inside chondrules measured by Tachibana and Huss (2005). Scale bars are $10 \mu \mathrm{m}$. (a) Putative primary troilite enclosed in an $\mathrm{Fe}-\mathrm{Ni}$ metal spherule within a chondrule, as indicated in Figure 1c. (b) Putative primary troilite enclosed in an $\mathrm{Fe}-\mathrm{Ni}$ metal spherule within a chondrule, as indicated in Figure 1d. The inset shows an enlarged image of the troilite. (c) Troilite-metal assemblage in a chondrule, as indicated in Figure 1e.

As sulfur is a volatile element, it would have evaporated either from silicate melt or from $\mathrm{Fe}^{-} \mathrm{S}$ melt, immiscible with silicate melt, at chondrule-forming temperatures (e.g., Yu et al., 1996). During cooling, the sulfur would have recondensed onto chondrules as sulfide veneers or as opaque assemblages such as those found in the matrix or at chondrule rims (e.g., Zanda et al., 1995; Lauretta et al., 2001; Lauretta and Buseck, 2003). Thus, troilites inside and outside of chondrules might record evaporation during heating, and condensation during cooling. Tachibana and Huss (2005) first attempted to find evidence of isotopic fractionation of sulfur due to kinetic evaporation during chondrule formation, as it was known that isotopic mass fractionation of sulfur occurs during evaporation into a surrounding low-pressure atmosphere (McEwing et al., 1980; Tachibana and Tsuchiyama, 1998a; Tachibana et al., unpublished). As sulfur is a mobile element in the parent body and may form secondary troilites on the parent body, Tachibana and Huss (2005) carefully examined troilites within chondrules to find those that crystallized from $\mathrm{Fe}-\mathrm{S}$ melt but which had not evaporated completely during chondrule formation. They then defined troilites enclosed in metallic $\mathrm{Fe}-\mathrm{Ni}$ spherules as primary troilites (Fig. 2a, b) because they are located inside solid metal spherules that were molten during chondrule formation and are difficult to form from parent-body processes. Such troilites are found only in large metallic spherules within volatile-poor type I chondrules, indicating that the sulfides crystallized from $\mathrm{Fe}-\mathrm{S}$ melts that retained sulfur from the chondrule precursors due to their small surfaceto-volume ratios. The authors did not observe any sulfide trails to the sulfide-enclosing metallic spherules that formed from sulfur mobilization during parent-body metamorphism. Several lines of evidence therefore suggest that troilites enclosed in metallic spherules are primary in nature and survived chondrule-forming high-temperature events. The presence of primary troilites in chondrules indicates that the chondrule formation event began with chondrule precursors at an ambient temperature low enough to retain sulfur, i.e., $<700 \mathrm{~K}$ for the system of solar abundance.

Ion microprobe analyses reveal no significant differences in the sulfur isotopic compositions of chondrule troilites and troilites outside of chondrules (Fig. 3). The largest measured fractionation was $2.7 \pm 1.4 \%$ o $/ \mathrm{amu}$ (atomic mass unit), while all other troilite grains recorded isotopic fractionations of $<1 \%$ omu. These suppressed sulfur isotopic fractionations place constraints on the evaporation behavior of sulfur during chondrule formation.

Recondensation of evaporated gases during the cooling of chondrules may explain the lack of isotopic fractionation of potassium and other lithophile elements within chondrules (e.g., Nagahara and Ozawa, 2000; Ozawa and Nagahara, 2001). However, as mentioned above, sulfur would most likely recondense into a physically distinct reservoir (for instance, thick sulfide rims 


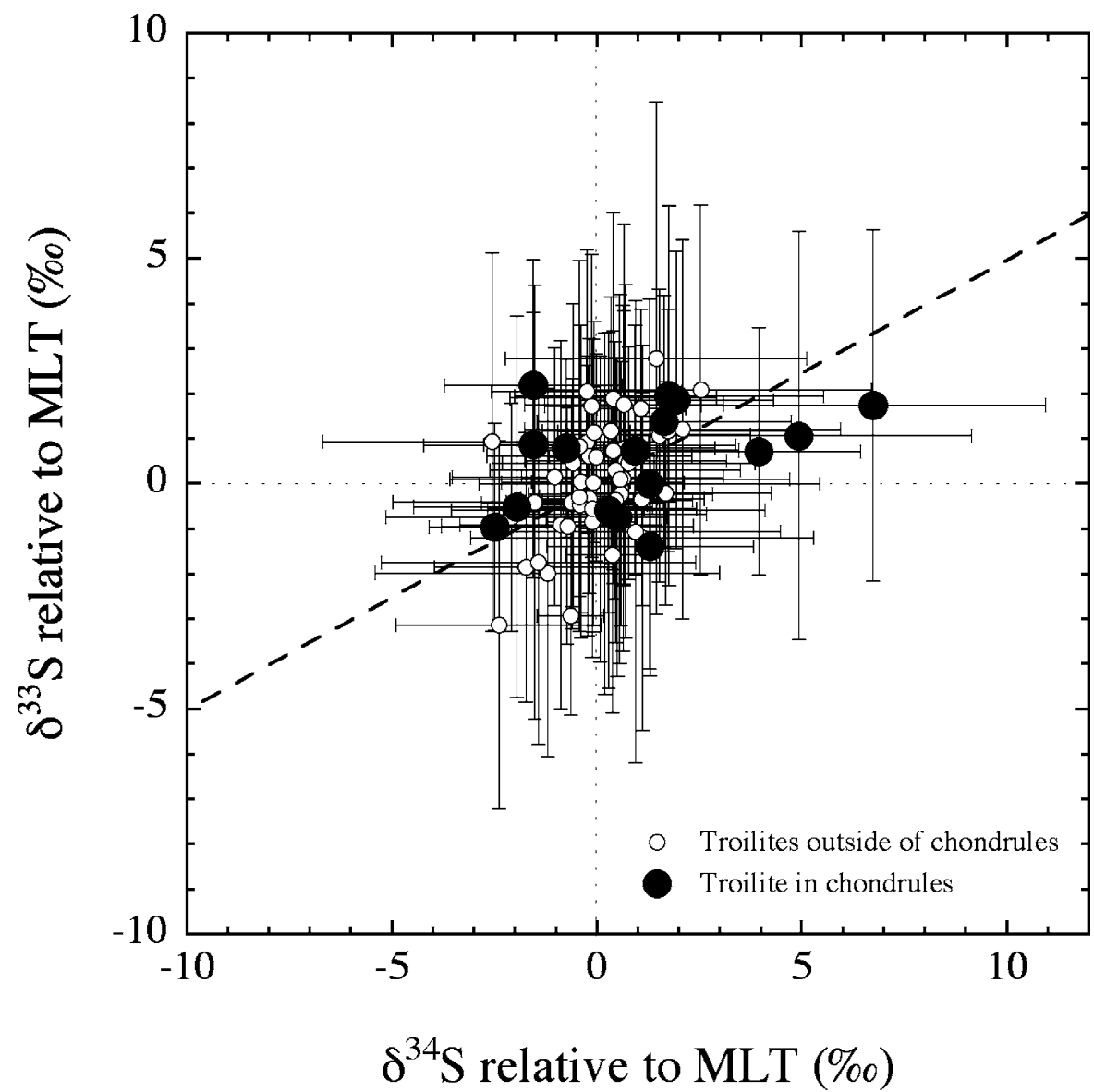

Figure 3. Isotopic compositions of sulfur in troilite grains within chondrules from Semarkona (LL3.0) and Bishunpur (LL3.1) relative to the mean sulfur isotopic composition of large troilites in the matrix (MLT). Data are from Tachibana and Huss (2005). $\delta^{\mathrm{m}} \mathrm{S}$ $(\% \circ)$ is defined as $\left\{\left[\left({ }^{\mathrm{m}} \mathrm{S}{ }^{\beta 2} \mathrm{~S}\right)_{\text {chondrule }} /\right.\right.$ $\left.\left.\left({ }^{\mathrm{m}} \mathrm{S} /{ }^{32} \mathrm{~S}\right)_{\mathrm{MLT}}\right]-1\right\} \times 1000(\mathrm{~m}=33,34)$. Error bars are $2 \sigma$. around chondrules) that was not in communication with the primary troilites; therefore, recondensation probably does not explain the lack of isotopic fractionation in primary troilites. Evaporation in the presence of a back reaction might also suppress sulfur isotopic fractionation. However, extremely highly dust-enriched conditions, in which other elements would not evaporate, would be required for sulfur due to its high volatility. Although boiling of the $\mathrm{Fe}-\mathrm{S}$ melt is another way to suppress fractionation, this is inconsistent with experimental observations (Tachibana and Tsuchiyama, 1998a). Evaporation of sulfur through a surrounding silicate melt most likely explains the decoupling of chemical and isotopic fractionations, however, this mechanism only occurs at temperatures above the solidus of silicate grains. To explain the observations, isotopic fractionation should also be suppressed before silicate grains begin to melt. Thus Tachibana and Huss (2005) modeled the evaporation of troilite and $\mathrm{Fe}^{-} \mathrm{S}$ melt and accompanying sulfur isotopic fractionation in a $\mathrm{Fe}-\mathrm{S}-\mathrm{H}$ system that is open for gas-solid (or gas-liquid) reactions but closed for the abundance of elements based on evaporation kinetics of sulfur from troilite (Tachibana and Tsuchiyama, 1998b). Their kinetic evaporation model suggests that a rapid heating rate of $>10^{4}$
$\mathrm{K} / \mathrm{h}$ for a temperature range of $1300-1600 \mathrm{~K}$, where significant silicate melting does not occur, is required to explain observed sulfur isotopic fractionations. This is the first quantitative estimate of the heating rate of chondrule precursors; the estimated rate is much faster than the cooling rates of chondrule melt.

\section{Plausible thermal history of chondrules}

I propose the following plausible thermal history of chondrules. (i) The chondrule formation event began with chondrule precursors at an ambient temperature low enough to retain volatiles. (ii) With the heating of chondrule precursors, evaporation of volatile elements, including sulfur, occurred from solid precursors. (iii) Once the temperature of precursors reached the $\mathrm{Fe}-\mathrm{S}$ eutectic temperature $(1261 \mathrm{~K})$, evaporation of sulfur occurred from the $\mathrm{Fe}^{-} \mathrm{S}$ melt. The heating rate $\left(>10^{4} \mathrm{~K} / \mathrm{h}\right)$ was sufficiently rapid to suppress sulfur evaporation $(<20 \%)$ and accompanying isotopic fractionation ( $<3 \%$ olamu). (iv) At higher temperatures, after silicate precursors had melted and surrounded the immiscible $\mathrm{Fe}^{-} \mathrm{S}$ melt, sulfur evaporation occurred through the silicate melt, and some sulfidemetal melt droplets were expelled from the chondrules. 
This stage would have produced variations in the abundance of sulfur within chondrules without generating a significant degree of isotopic fractionation. Evaporation of volatile lithophile elements such as $\mathrm{Na}$ and $\mathrm{K}$ also occurred from silicate melts. The presence of primary troilites and alkali elements in chondrules suggests that the duration of heating at temperatures close to the peak temperature might not have been long enough for the complete loss of volatiles from the melt. (v) During cooling of chondrules at a rate of $\sim 5-1000 \mathrm{~K} / \mathrm{h}$, evaporated volatile lithophile elements began to recondense into chondrules, and isotopic equilibration of elements such as potassium may have occurred. (vi) Sulfur then began to recondense to form opaque assemblages, forming different sulfur reservoirs from troilites inside chondrules, via reactions with metal grains at the rim or outside of chondrule spherules. Note that sulfur would also have evaporated from the expelled $\mathrm{Fe}-\mathrm{S}$ melt during stages (iv) and (v), which may have led to the enrichment of heavier sulfur isotopes in the expelled melt. However, evaporated sulfur would have recondensed onto the expelled metalsulfide to diminish the record of isotopic fractionation, as in the case of the recondensation of volatile lithophile elements onto the chondrules.

\section{HEATING MECHANISMS AND SITE OF CHONDRULE FORMATION}

Several heat sources have been proposed for chondrule formation, including nebular lightning, shock waves, $\mathrm{X}^{-}$ wind, and impact; the advantages and disadvantages of these sources are reviewed by Boss (1996) and Jones et al. (2000). Given the current state of knowledge, shockwave heating is one of the most plausible models for chondrule formation, as it explains various properties of chondrules, including the peak temperatures and cooling rates described above (Desch et al., 2005 and references therein). Here we compare the newly proposed constraint on the heating rates of chondrules $\left(>10^{4} \mathrm{~K} / \mathrm{h}\right.$; Tachibana and Huss, 2005) with rates predicted from shock-wave models.

In the case of shock-wave heating, chondrule precursors in the preshock region (i.e., before the passing of the shock front) are heated by radiation from the postshock region, while in the postshock region they are heated by gas drag. Gas-drag heating is strong enough to melt solid grains and form chondrules for appropriate shock speeds and gas densities. The heating/cooling rate of precursors in the postshock region is determined by energy transfer between gas and dust, radiative cooling of dust, and latent heat cooling due to the evaporation of dust or melt.
Miura and Nakamoto (2005) numerically simulated shock-wave heating of chondrules to find appropriate shock conditions to satisfy the heating and cooling rates determined from natural chondrules. They found that radiative heating in the preshock region is effective in the case of heating in a high gas density region $\left(\sim 10^{20}\right.$ particles $\left./ \mathrm{m}^{3}\right)$ with low shock velocities $(\sim 10 \mathrm{~km} / \mathrm{s})$, and that the heating rate of precursors in the temperature range of $1300-1600 \mathrm{~K}$ could be $<10^{3} \mathrm{~K} / \mathrm{h}$, which is inconsistent with the constraints proposed by Tachibana and Huss (2005). In contrast, for low gas density conditions $\left(<10^{19}\right.$ particles $/ \mathrm{m}^{3}$ ) with high shock velocities $(>20 \mathrm{~km} / \mathrm{s}$ ), both heating and cooling rates agree with the ranges predicted from natural chondrules, which may constrain the source of chondrule-forming shock waves.

The disadvantage of shock heating models is that the source of the shock wave is uncertain. Many mechanisms for shock waves have been proposed, such as bow shocks in front of fast-moving planetesimals, accretion shocks at the surface of the protoplanetary disk, and spiral density waves induced by gravitational instability of the disk (Boss, 1996; Nakamoto et al., 2005b and references therein).

As discussed above, several constraints have been proposed on the timing and duration of chondrule-forming events and on the thermal history of chondrules during each heating event. Of the proposed shock sources listed above, gravitational instability of the protoplanetary disk appears inadequate to explain the constraints of chondrule formation because it is unlikely that spiral density waves occur repeatedly over a period of a few million years in the protoplanetary disk, and because the high gas density required for gravitational instability is inconsistent with rapid heating rates. Accretion shocks for material that infalls onto the surface of the protoplanetary disk are also unlikely because chondrules were heated only once when dust particles accreted onto the disk. Bow shocks in front of fast-moving planetesimals might explain the constraints for chondrule formation as long as Jupiter formed during the very early stages of disk evolution.

Another source recently proposed for shock waves is the interaction of a strong gas flow, generated by $\mathrm{X}$-ray flares during the T-Tauri stage of the young Sun, and disk material in the upper region of the protoplanetary disk (Nakamoto et al., 2005b; see also Fig. 4 in Hayashi et al., 1996). X-ray flares are commonly found among T-Tauri stars, with intensities $\sim 100$ times larger than those from the present Sun (Feigelson et al., 2002). Events lasted for 1-10 Myr throughout the T-Tauri stage (Feigelson et al., 2002), which is consistent with the timing and duration of chondrule-forming events. High-temperature plasma gas 
cools to a strong neutral gas wind that flows upward along the rotation axis of the central star, but part of the gas flow may have hit the surface of the protoplanetary disk and generated shock waves.

Nakamoto et al. (2005b) numerically simulated $\mathrm{X}^{-}$ ray flares and accompanying strong neutral gas flow using a 2-D magnetohydrodynamics code (Hayashi et al., 1996), and examined interaction of the gas flow with the protoplanetary disk. They found that part of a strong neutral gas wind hits the surface of the protoplanetary disk and generates shock waves in the upper region of the protoplanetary disk at 1-1.5 AU via interaction with the disk gas. High-speed shock waves in the low-density gas region of the disk are strong enough to melt chondrule precursors. Such shock conditions are consistent with the shock conditions that satisfy the heating and cooling histories of chondrules (Miura and Nakamoto, 2005). Although further careful investigations are needed, the model that involves shock-wave heating induced by $\mathrm{X}^{-}$ ray flares appears to be a plausible model for chondrule formation.

\section{CONCLUSIONS}

In this study I reviewed the nature of localized transient high-temperature events that formed chondrules in the early solar system at $\sim 4.57 \mathrm{Ga}$. This review is based on recent progress in the field of planetary material science, including the timing and duration of the chondrule-forming period, the thermal history of chondrules during each heating event, heating mechanisms, and sites where such high-temperature heating occurred.

(1) Long-lived and short-lived chronometers show that the chondrule formation period began shortly after or contemporaneous with CAI formation $(\sim 4.567$ $\mathrm{Ga})$ and lasted for a few million years. The duration of the chondrule formation period is consistent with that for the classical T-Tauri stage of young lowmass Sun-like stars, which corresponds to the active energetic stage of the protoplanetary disk.

(2) Dynamic crystallization experiments reveal that chondrule melt droplets were heated to 1800-2200 $\mathrm{K}$ for short durations and cooled rapidly at rates of $~$ 5-1000 K/h. The isotopic compositions of sulfur from chondrule troilites and the evaporation model of sulfur constrain the heating rate of chondrule precursors to $>10^{4} \mathrm{~K} / \mathrm{h}$ in the temperature range $1300^{-}$ $1600 \mathrm{~K}$.

(3) One of the plausible heating mechanisms for chondrules is shock-wave heating. Detailed simulations of shock-wave heating with various shock parameters demonstrate that high-velocity shock waves in low-pressure regions may be appropriate for the estimated thermal history of chondrules.

(4) Many sources have been proposed for shock waves in the protoplanetary disk. X-ray flaring of young stars, which is commonly observed for $\mathrm{T}$-Tauri stars and continues for 1-10 Myr, is one of the recently proposed sources for shock waves. The interaction of strong gas flow induced by $\mathrm{X}$-ray flare with the upper region of the protoplanetary disk can produce shock waves that are strong enough to produce chondrules; this may satisfy existing constraints on chondrule formation.

\section{ACKNOWLEDGMENTS}

I thank K. Tomeoka, A. Tsuchiyama and H. Yurimoto, who organized the 2004 Symposium of the Mineralogical Society of Japan "The frontiers of science for primitive solar system materials; The role of material science", for inviting me to give a talk at the symposium and for giving me the opportunity to write this review. Constructive reviews by $\mathrm{H}$. Yurimoto and an anonymous reviewer, and a helpful review and editorial assistance by $\mathrm{K}$. Tomeoka are greatly appreciated. The section on chronology was originally planned to be reviewed by N. T. Kita, who has intensively studied ${ }^{26} \mathrm{Al}-{ }^{26} \mathrm{Mg}$ systems in ferromagnesian chondrules in recent years. I am indebted to G.R. Huss and N.T. Kita for valuable discussions and their help in ion microprobe analyses, to A. Tsuchiyama, H. Nagahara, and K. Ozawa for fruitful discussions and support with experimental and theoretical studies of kinetic evaporation and condensation, and to T. Nakamoto and H. Miura for helping me understand shock-wave heating mechanisms.

\section{REFERENCES}

Amelin, Y., Krot, A.N., Hutcheon, I.D. and Ulyanov, A.A. (2002) Lead isotopic ages of chondrules and calcium-aluminumrich inclusions. Science, 297, 1678-1683.

Amelin Y., Krot A. and Twelker E. (2004) Pb isotopic age of the $\mathrm{CB}$ chondrite Gujba, and the duration of the chondrule formation interval. Geochimica et Cosmochimica Acta, 68, A759.

Amelin, Y. and Krot, A.N. (2005) Young Pb-isotopic ages of chondrules in $\mathrm{CB}$ carbonaceous chondrites. Lunar and Planetary Science, 36, 1247.

Bizzarro, M., Baker, J.A. and Haack, H. (2004) Mg isotope evidence for contemporaneous formation of chondrules and refractory inclusions. Nature, 431, 275-278.

Boss, A.P. (1996) A concise guide to chondrule formation models. In Chondrules and the Protoplanetary Disk (Hewins, R.H. et al., Eds.). Cambridge University Press, Cambridge, U.K., 257-263.

Chaussidon, M., Bobert, F. and McKeegan, K. D. (2002) Incor- 
poration of short-lived ${ }^{7} \mathrm{Be}$ in one $\mathrm{CAI}$ from the Alende meteorite. Lunar and Planetary Science, 33, 1563.

Desch, S.J. and Connolly, H.C., Jr. (2002) A model of the thermal processing of particles in solar nebula shocks: Application to the cooling rates of chondrules. Meteoritics \& Planetary Science, 37, 183-207.

Desch, S.J., Ciesla, F.J., Hood, L.L. and Nakamoto, T. (2005) Shock heating; Effecs on chondritic material. In Chondrites and the Protoplanetary Disk (Krot, A.E., Scott E.R.D. and Reipurth B. Eds.). Astronomical Society of the Pacific, 849872.

Feigelson, E.D., Garmire, G.P. and Pravdo, S.H. (2002) Magnetic flaring in the pre-main-sequence sun and implications for the early solar system. Astrophysical Journal, 572, 335-349.

Galy, A., Young, E.D., Ash, R.D. and O'Nions, R.K. (2000) The formation of chondrules at high gas pressures in the solar nebula. Science, 290, 1751-1754.

Galy, A., Hutcheon, I.D. and Grossman, L. (2004) $\left({ }^{26} \mathrm{Al} /{ }^{27} \mathrm{Al}\right)_{0}$ of the solar nebula inferred from $\mathrm{Al}-\mathrm{Mg}$ systematic in bulk CAIs from CV 3 chondrites. Lunar and Planetary Science, 35, 1790.

Hayashi, M.R., Shibata, K., and Matsumoto, R. (1996) X-ray flares and mass outflows driven by magnetic interaction between a protostar and its surrounding disk. Astrophysical Journal Letters. 468, L37-L41.

Hewins, R.H. (1988) Experimental studies of chondrules. In Meteorites and the Early Solar System (Kerridge, J.F. and Mathews, M.S., Eds.). University of Arizona Press, Tucson, USA, 660-679.

Hewins, R.H. and Connolly, H.C., Jr. (1996) Peak temperatures of flash-melted chondrules. In Chondrules and the Protoplanetary Disk (Hewins, R.H. et al., Eds.). Cambridge University Press, Cambridge, U.K., 197-204.

Huss, G.R., MacPherson, G.J., Wasserburg, G.J., Russell, S.S. and Srinivasan, G. (2001) Aluminum-26 in calcium-aluminumrich inclusions and chondrules from unequilibrated ordinary chondrites. Meteoritics \& Planetary Science, 36, 975-997.

Itoh, S. and Yurimoto H. (2003) Contemporaneous formation of chondrules and refractory inclusions in the early Solar System. Nature, 423, 728-731.

Jones, R.H. (1996) Relict grains in chondrules: Evidence for chondrule recycling. In Chondrules and the Protoplanetary Disk (Hewins, R.H. et al., Eds.), Cambridge University Press, Cambridge, U.K., 164-172.

Jones, R.H., Lee, T., Connolly, H.C., Jr., Love, S.G. and Shang, H. (2000) Formation of chondrules and CAIs: Theory vs. observation. In Protostars and Planets IV (Mannings, V. et al., Eds.). University of Arizona Press, Tucson, USA, 927-962.

Kita, N.T., Nagahara, H., Togashi, S. and Morishita, Y. (2000) A short duration of chondrule formation in the solar nebula: evidence from ${ }^{26} \mathrm{Al}$ in Semarkona ferromagnesian chondrules. Geochimica et Cosmochimica Acta, 64, 3913-3922.

Kita, N.T., Huss, G.R., Tachibana, S., Amelin, Y., Nyquist, L.E. and Hucheon, I.D. (2005a) Constraints on the Origin of Chondrules and CAIs from Short-Lived and Long-Lived Radionuclides. In Chondrites and the Protoplanetary Disk (Krot, A.E., Scott E.R.D. and Reipurth B. Eds.). Astronomical Society of the Pacific, 558-587.

Kita, N.T., Tomomura, S., Tachibana, S., Nagahara, H., Mostefaoui, S. and Morishita, Y. (2005b) Correlation between aluminum-26 ages and bulk $\mathrm{Si} / \mathrm{Mg}$ ratios for chondrules from LL3.0-3.1 chondrites. Lunar and Planetary Science, 36,
1750.

Krot, A.N., Zolensky, M.E., Wasson, J.T., Scott, E.R.D., Keil, K. and Ohsumi, K. (1997) Carbide-magnetite assemblages in type-3 ordinary chondrites. Geochimica et Cosmochimica Acta, 61, 219-237.

Krot, A.N., Yurimoto, H., Hutcheon, I,D. and MacPherson, G.J. (2005) Chronology of the early Solar System from chondrule-bearing calcium-aluminium-rich inclusions. Nature, 434, 998-1001.

Kunihiro, T., Rubin, A.E., McKeegan, K.D. and Wasson, J. T. (2004) Initial ${ }^{26} \mathrm{Al} /{ }^{27} \mathrm{Al}$ in carbonaceous-chondrite chondrules: too little ${ }^{26} \mathrm{Al}$ to melt asteroids. Geochimica et Cosmochimica Acta, 68, 2947-2957.

Kurahashi, E., Kita, N.T., Nagahara, H. and Morishita, Y. (2004) Contemporaneous formation of chondrules in the ${ }^{26} \mathrm{Al}-{ }^{26} \mathrm{Mg}$ system for ordinary and $\mathrm{CO}$ chondrites. Lunar and Planetary Science, 35, 1476.

Lauretta, D.S., Buseck, P.R. and Zega, T.J. (2001) Opaque minerals in the matrix of the Bishunpur (LL3.1) chondrite: constraints on the chondrule formation environment. Geochimica et Cosmochimica Acta, 65, 1337-1353.

Lauretta, D.S. and Bueck, P.R. (2003) Opaque minerals in chondrules and fine-grained chondrule rims in the Bishupur (LL3.1) chondrite. Meteoritics \& Planetary Science, 38, 5979.

Lee, T., Papanastassiou, D.A. and Wasserburg, G.J. (1976) Demonstration of ${ }^{26} \mathrm{Mg}$ excess in Allende and evidence for ${ }^{26} \mathrm{Al}$. Geophysical Research. Letters, 3, 109-112.

Lofgren, G.E. (1996) A dynamic crystallization model for chondrule melts. In Chondrules and the Protoplanetary Disk (Hewins, R.H. et al., Eds.). Cambridge University Press, Cambridge, U.K., 187-196.

MacPherson, G.J., Davis, A.M. and Zinner, E.K. (1995) The distribution of ${ }^{26} \mathrm{Al}$ in the early solar system- a reappraisal. Meteoritics, 30, 365-386.

McEwing, C.E., Thode, H.G. and Rees, C.E. (1980) Sulphur isotope effects in the dissociation and evaporation of troilite: A possible mechanism for ${ }^{34} \mathrm{~S}$ enrichment in lunar soils. Geochimica et Cosmochimica Acta, 44, 565-571.

McKeegan, K.D., Greenwood, J.P., Leshin, L.A. and Cosarinsky, M. (2000) Abundance of ${ }^{26} \mathrm{Al}$ in ferromagnesian chondrules of unequilibrated ordinary chondrites. Lunar and Planetary Science, 31, 2009.

McKeegan, K.D. and Davis, A.M. (2003) Early solar system chronology. In Meteorites, Planets, and Comets (Davis, A.M. Ed.), Vol. 1 Treatise on Geochemistry (Holland, H.D. and Turekian, K. K. Eds.). Elsevier-Pergamon, Oxford, 431-460.

Miura, H. and Nakamoto, T. (2005) Appropriate shock waves for chondrule formation: heating rate and cooling rate constraints. Lunar and Planetary Science, 36, 1248.

Mostefaoui, S., Kita, N.T., Togashi, S., Tachibana, S., Nagahara, H. and Morishita, Y. (2002) The relative formation ages of ferromagnesian chondrules inferred from their initial ${ }^{26} \mathrm{Al} /{ }^{27} \mathrm{Al}$. Meteoritics \& Planetary Science, 37, 421-438.

Nagahara, H. (1981) Evidence for secondary origin of chondrules. Nature, 292, 135-136.

Nagahara, H. and Ozawa, K. (2000) Isotopic fractionation as a probe of heating processes in the solar nebula. Chemical Geology, 169, 45-68.

Nakamoto, T., Kita, N. T. and Tachibana, S. (2005a) Chondrule age distribution and rate of heating events for chondrule formation. Antarctic Meteorite Research, 18, 253-272. 
Nakamoto, T., Hayashi, M.R., Kita, N.T. and Tachibana, S. (2005b) Chondrule forming shock waves in solar nebula by $\mathrm{X}$-ray flares. In Chondrites and the Protoplanetary Disk (Krot, A.E. et al., Eds.). Astronomical Society of the Pacific, 883-892.

Ozawa, K. and Nagahara, H. (2001) Chemical and isotopic fractionations by evaporation and their cosmochemical implications. Geochimica et Cosmochimica Acta, 65, 2171-2199.

Rambaldi, E.R. (1981) Relict grains in chondrules. Nature, 293, 558-561.

Rubin, A.E., Kallemeyn, G.W., Wasson, J.T., Clayton, R.N., Mayeda, T.K., Grady, M., Verchovsky, A.B., Eugster, O. and Lorenzetti, S. (2003) Formation of metal and silicate globules in Gujba: a new Bencubbin-like meteorite fall. Geochimica et Cosmochimica Acta, 67, 3283-3298.

Russell, S.S., Srinivasan, G., Huss, G.R., Wasserburg, G.J. and MacPherson, G.J. (1996) Evidence for widespread ${ }^{26} \mathrm{Al}$ in the solar nebula and constraints for nebula time scales. Science, 273, 757-762.

Tachibana, S. and Tsuchiyama, A. (1998a) Measurements of evaporation rates of sulfur from iron-iron sulfide melt. Meteoritics \& Planetary Science, A153.

Tachibana, S. and Tsuchiyama, A. (1998b) Incongruent evaporation of troilite $(\mathrm{FeS})$ in the primordial solar nebula; an experimental study. Geochimica et Cosmochimica Acta, 62, 20052022.

Tachibana, S., Nagahara, H., Mostefaoui, S. and Kita, N.T. (2003) Correlation between relative ages inferred from ${ }^{26} \mathrm{Al}$ and bulk compositions of ferromagnesian chondrules in least equilibrated ordinary chondrites. Meteoritics \& Planetary Science, 38, 939-962.

Tachibana, S. and Huss, G. R. (2005) Sulfur isotope composition of putative primary troilite in chondrules from Bishunpur and Semarkona. Geochimica et Cosmochimica Acta, 69, 30753097.

Tachibana, S., Huss, G.R., Kita, N.T., Shimoda, G. and Morishita, Y. (2006) Iron-60 in chondrites: Debris from a nearby super- nova in the early solar system? Astrophysical Journal Letters, 639, L87-L90.

Tomomura, S., Nagahara, H., Tachibana, S., Kita, N.T. and Morishita, Y. (2004) Relationship between bulk chemical composition and formation age of chondrules in Bishunpur and Krymka. Lunar and Planetary Science, 35, 1555.

Tsuchiyama, A., Nagahara, H. and Kushiro, I. (1980) Experimental reproduction of textures of chondrules. Earth and Planetary Science Letters, 48, 155-165.

Tsuchiyama, A., Nagahara, H. and Kushiro, I. (1981) Volatilization of sodium from silicate melt spheres and its application to the formation of chondrules. Geochimica et Cosmochimica Acta, 45, 1357-1367.

Young, E.D., Simon, J.I., Galy, A., Russell, S.S., Tonui, E. and Lovera, O. (2005) Supra-canonical ${ }^{26} \mathrm{Al} /{ }^{27} \mathrm{Al}$ and the residence time of CAIs in the solar protoplanetary disk. Science, 5719, 223-227.

Yu, Y., Hewins, R.H. and Zanda, B. (1996) Sodium and sulfur in chondrules: heating time and cooling curves. In Chondrules and the Protoplanetary Disk (Hewins, R.H. et al., Eds.). Cambridge University Press, Cambridge, U.K., 213-219.

Yu, Y. and Hewins, R.H. (1998) Transient heating and chondrule formation: Evidence from sodium loss in flash heating simulation experiments. Geochimica et Cosmochimica Acta, 62, 159-172.

Yurimoto, H. and Wasson, J.T. (2002) Extremely rapid cooling of a carbonaceous-chondrite chondrule containing very ${ }^{16} \mathrm{O}^{-}$ rich olivine and ${ }^{26} \mathrm{Mg}$-excess. Geochimica et Cosmochimica Acta. $66,4355-4363$.

Zanda, B., Bourot-Denise, M. and Hewins, R.H. (1995) Condensate sulfide and its metamorphic transformations in primitive chondrites (abstract). Meteoritics, 30, 605.

Manuscript received March 22, 2005

Manuscript accepted December 7, 2005

Manuscript handled by Kazushige Tomeoka 